\title{
Challenges in Cancer Control Services Provided by Family Physicians in Primary Care: A Qualitative and Quantitative Study From Karabuk Province in Turkey
}

\author{
Raziye Özdemir ${ }^{1}$, Sevda Ural ${ }^{2, *}$, Merve Karaçalı ${ }^{3}$ \\ Departments of ${ }^{1}$ Occupational Health and Safety, ${ }^{2}$ Nursing, Karabuk University Faculty of Health Sciences, Demir Çelik Campus, ${ }^{3}$ Eflani Community \\ Health Center, Karabuk, Turkey
}

\begin{abstract}
Background: Family physicians (FPs) play an important role in cancer control. The aim of this study was to understand the functions of FPs in cancer control and to explore FPs' perceptions of their own roles and the difficulties they face in cancer control in Karabuk province, Turkey.

Methods: The study consisted of two methodological parts. The qualitative part included a descriptive study in which data were collected from $87.5 \%$ ( $n=56$ ) out of all FPs in Karabuk using a questionnaire. In the quantitative part, in-depth interviews with 15 FPs were conducted and analyzed through content analysis.

Results: Half of the FPs (50.0\%) provided cancer prevention information for their registered people, focusing on especially smoking cessation. In the last three months, the proportion of FPs who had not invited anyone to screenings was $37.5 \%$ for the pap test, $26.8 \%$ for the mammography, $19.0 \%$ for the fecal occult blood test and $34.5 \%$ for the colonoscopy. Only $16.1 \%$ of them reported that they made home visits for cancer patients. In the qualitative part of study, the following themes were highlighted: the perceived responsibilities of FPs regarding cancer control; the effect of geographically undefined working area of FPs; the issues with coordination between FPs and specialists; the effect of the number of primary care team members.
\end{abstract}

Conclusions: Cancer control services provided by FPs have significant problems in terms of the FPs' approach to the services and their content, continuity and coordination.

(J Cancer Prev 2018;23:176-182)

Key Words: Cancer, Family physicians, Primary care

\section{INTRODUCTION}

Cancer control requires comprehensive and continuous practices ranging from reducing the effects of risk factors at the individual and environmental levels as much as possible to maintaining care until the end of life. Family physicians (FPs) have critical roles in all stages of cancer control services as the first contact with the community.

Studies indicate that the services provided by FPs are associated with an increase in public awareness and in the early diagnosis of cancer $^{1-3}$ and with improvements in survival duration and quality of life of cancer patients, the provision of end-of-life care, and allowing end-stage cancer patients to die at home. ${ }^{48}$ The need for primary, secondary and tertiary care providers to share the burden of follow-up care for the increasing number of cancer patients has also been noted. ${ }^{9.10}$ The Turkish National Cancer Control Program highlighted the integration of FPs into the cancer control is a necessity for the success of cancer control program. ${ }^{11}$ In addition, the FPs should provide the primary and secondary prevention of cancer, monitoring of patients with cancer and other chronic diseases and meeting those patients' home care needs legally. However, there have

Received June 28, 2018, Revised September 23, 2018, Accepted September 27, 2018

Correspondence to: Raziye Özdemir

Department of Occupational Health and Safety, Karabuk University, Demir Celik Kampus, 78050 Karabuk, Turkey

Tel: +90-542-5660818, Fax: +90-370-433-33-27, E-mail: raziyeozdemir@karabuk.edu.tr, ORCID: Raziye Özdemir, https://orcid.org/0000-0002-7033-3471

*Current affiliation: Department of Occupational Health and Safety, Akdagmadeni School of Health, Bozok University, Yozgat, Turkey.

Copyright (C) 2018 Korean Society of Cancer Prevention

(c) This is an Open Access article distributed under the terms of the Creative Commons Attribution Non-Commercial License (http://creativecommons.org/icenses/by-nc/4.0) which permits unrestricted non-commercial use, distribution, and reproduction in any medium, provided the original work is properly cited. 
been no studies of the role of FPs in cancer control in Turkey based on our best knowledge.

The aim of this study was to evaluate the functions of FPs in cancer control and to explore FPs' perceptions of their own roles and the difficulties they face in cancer control in Karabuk province, Turkey.

\section{MATERIALS AND METHODS}

The study was carried out in Karabuk located in northwest Turkey in 2014 to 2015 years. The study has two methodological parts (Fig. 1).

\section{The qualitative part of the study}

A descriptive study was established. The study group consisted of $56(87.5 \%)$ of a total of 64 FPs (six physicians refused to participate in the study, and two physicians were not available because they were off duty). Data were collected through face-to-face interviews using a questionnaire based on the relevant literature. The questionnaire included 35 questions that questioned physicians' sociodemographic and work-related characteristics and functions in cancer control services. Descriptive data were summarized as a percentage distribution.

\section{The quantitative part of the study}

A descriptive qualitative approach was adopted in this part of study. In-depth interviews were conducted with 15 FPs by using an interview guide. The interview guide included open-ended questions such as "1) could you please inform about your services in primary care settings related to cancer prevention, early detection and the monitoring of patients with cancer? 2) could you explain about the factors that affect the quality of your services related to cancer control? 3) to what extent are you able to keep informed of the services your patients with cancer received from specialists and the results of their treatments?" Interviews were conducted until no new information was emerging, to saturation point. Each interview took 25 to 45 minutes and was tape-recorded after an oral consent obtained from each FP. The content analysis was used in the analysis of the data. First, the interviews were transcribed verbatim by researchers. Second, each researcher analyzed the data independently. Meaning units were identified for each interview, and then they were condensed and coded. The codes were compared to find similarities and differences. Afterwards, suitable subcategories and categories were formed by grouping together overlapping or similar codes. The themes were discussed until a consensus was reached and then demonstrated.

Ethics committee approval was obtained from the Bulent Ecevit University Research Ethics Committee (no: 2014-70-25-03).

\section{RESULTS}

\section{The qualitative part of the study}

Most of the FPs (67.9\%) were male and between the ages of 35 and 44 years $(76.8 \%)$, and $58.9 \%$ of the FPs worked in the city center. The majority of the physicians (91.1\%) were general

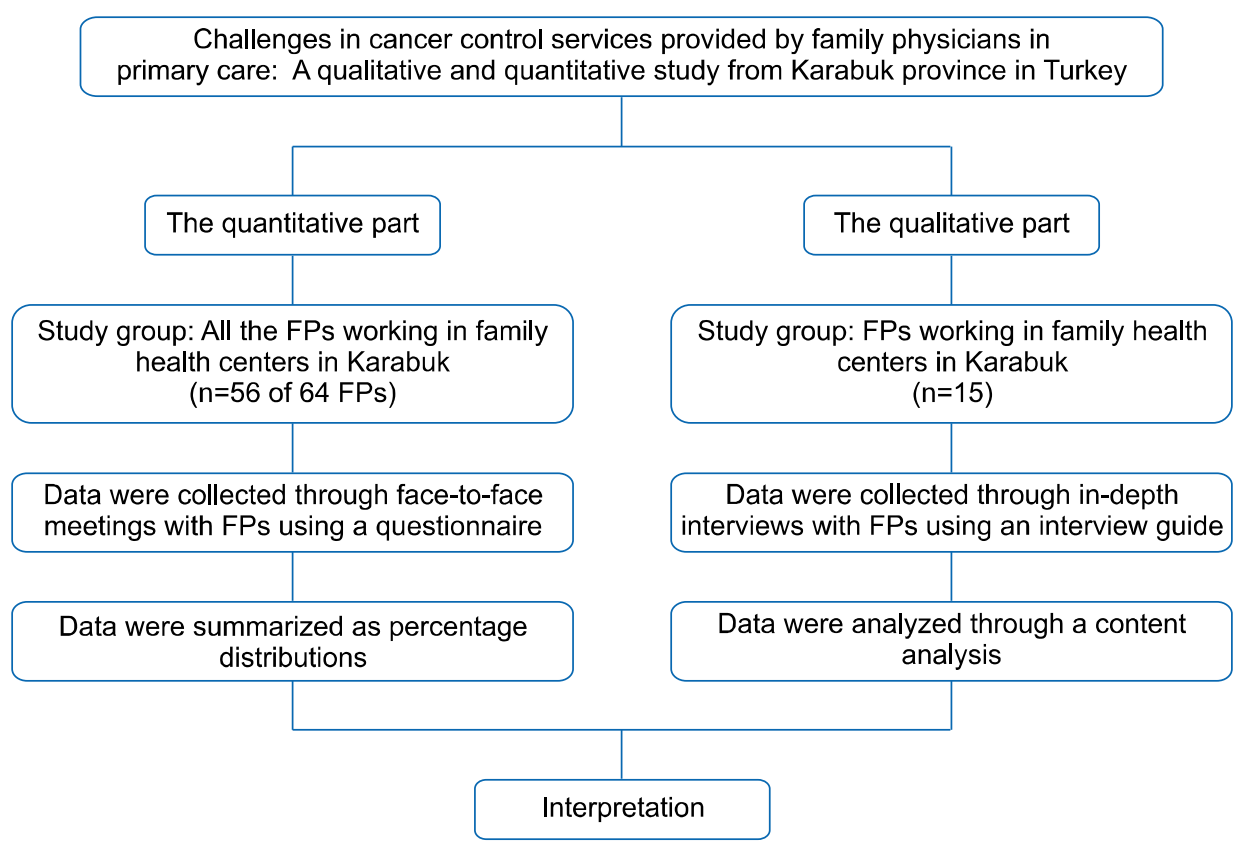

Figure 1. The design of the study. FP, family physicians. 
practitioners, and approximately half the FPs (51.8\%) were responsible for 3,500 or more individuals (Table 1 ).

Half of the FPs (50.0\%) reported that they had the opportunity to provide health education regarding cancer prevention on a regular basis. The FPs' health education activities focused mostly on patients' smoking behavior. The proportions of FPs who had never recommended that target patients take a screening test in the last three months were as follows: $37.5 \%$ for pap test, $26.8 \%$ for mammography, $19.0 \%$ for fecal occult blood test, and $34.5 \%$ for colonoscopy (all of these tests are within the scope of the national community-based screening program). One out of every three FPs (32.1\%) did not know the number of cancer patients in their registered population. Only $16.1 \%$ of the FPs performed home visits for their cancer patients (Table 2).

Table 1. Sociodemographic and work-related characteristics of the study group

\begin{tabular}{|c|c|}
\hline Variable & Number (\%) \\
\hline \multicolumn{2}{|l|}{ Sex } \\
\hline Female & $18(32.1)$ \\
\hline Male & $38(67.9)$ \\
\hline \multicolumn{2}{|l|}{ Age group (yr) } \\
\hline $25-34$ & $5(8.9)$ \\
\hline $35-44$ & $43(76.8)$ \\
\hline $45-54$ & $8(14.3)$ \\
\hline \multicolumn{2}{|l|}{ Work place } \\
\hline City center & $33(58.9)$ \\
\hline District cenrer & $19(33.9)$ \\
\hline Town/village & $4(7.1)$ \\
\hline \multicolumn{2}{|l|}{ Speciality } \\
\hline General practitioner & $51(91.1)$ \\
\hline Family physician & $5(8.9)$ \\
\hline \multicolumn{2}{|l|}{ Years in profession } \\
\hline $1-10$ & $12(21.4)$ \\
\hline $11-20$ & $36(64.3)$ \\
\hline $21-30$ & $8(14.3)$ \\
\hline \multicolumn{2}{|c|}{ Years in the family medicine model } \\
\hline $1-4$ & $16(28.5)$ \\
\hline $5-8$ & $40(71.5)$ \\
\hline \multicolumn{2}{|c|}{ No. of registered people per FP } \\
\hline $1,500-2,499$ & $7(12.5)$ \\
\hline $2,500-3,499$ & $20(35.7)$ \\
\hline $3,500-4,499$ & $29(51.8)$ \\
\hline \multicolumn{2}{|c|}{ Average no. of patients per day } \\
\hline $20-39$ & $10(17.9)$ \\
\hline $40-59$ & $23(41.1)$ \\
\hline $60-79$ & $17(30.4)$ \\
\hline 80 and over & $6(10.7)$ \\
\hline Total & $56(100)$ \\
\hline
\end{tabular}

The sum of the percentages does not equal $100 \%$ because of rounding. FP, family physician.

\section{The qualitative part of the study}

In this part, four themes were identified from in-depth interviews with two female and 13 male FPs.

1) Perceived responsibilities of family physicians regarding cancer control

All the FPs described their responsibilities as health education and early detection activities, which included screening for breast, cervix, and colorectal cancers in collaboration with Cancer Early Diagnosis Screening and Training Centers.

"We are given responsibilities mainly for the screening part; it seems so. Apart from that, our responsibilities are not much."

— Interview 15

The physicians indicated that their function was limited to referring patients to specialists following a cancer diagnosis, and they did not feel responsible for the follow-up care of cancer patients. Eight of the FPs stated that the responsibility for providing follow-up and palliative care for cancer patients belonged to the home care unit, which has two ambulance teams. Five FPs noted that it was not possible for them to provide follow-up care for cancer patients because of their daily routines and responsibilities.

"When we identify cancer patients, we only register them on the computer, but we cannot monitor them. How can you achieve that when you have already an excessive workload?"

- Interview 10

2) The effect of geographically undefined working area of family physicians

Nine FPs indicated that their lists were not constant because of migration and the patients' right to change physicians. They also mentioned that they encountered people who were not registered at any FP. The FPs stated that because the patients on their lists lived in different places, they were not able to assess the patients' living and workplaces. Five of the FPs noted that they could not plan or evaluate services, including cancer control, at the family health center level because they did not serve a geographically defined region.

"The Ministry of Health may calculate statistics at the geographic area level, but I cannot do it here. For example, there is a recycling factory in Karabuk, and sometimes workers have 
Table 2. Cancer prevention and early detection activities, and services after diagnosis of cancer provided by FPs $(n=56)$

\begin{tabular}{|c|c|}
\hline Activity & Number (\%) \\
\hline \multicolumn{2}{|l|}{ Health education } \\
\hline $\begin{array}{l}\text { In general, I am able to give an information about cancer prevention to the people who consu } \\
\text { My health education on cancer prevention mainly covers the following topics: }\end{array}$ & $28(50.0)$ \\
\hline Harms of smoking & $47(83.9)$ \\
\hline Avoiding obesity & $44(78.6)$ \\
\hline Physical activity & $42(75.0)$ \\
\hline Healthy diet & $34(60.7)$ \\
\hline Occupational and environmental carcinogens & $22(39.3)$ \\
\hline Sexual factors associated with cancer & $19(33.9)$ \\
\hline \multicolumn{2}{|l|}{ Screening } \\
\hline \multicolumn{2}{|c|}{ In past three months, I have invited at least one person from my eligible target population to undergo the following screening tests ${ }^{a}$ : } \\
\hline Pap smear or HPV DNA test & $35(62.5)$ \\
\hline Mammography & $41(73.2)$ \\
\hline Fecal occult blood test & $45(80.4)$ \\
\hline Colonoscopy & $36(65.5)$ \\
\hline \multicolumn{2}{|l|}{ Services after diagnosis of cancer } \\
\hline I know the total number of cancer patients among my registered population. & $38(67.9)$ \\
\hline I know the medical history of my cancer patients. & $6(10.9)$ \\
\hline I know my patients' family members living in their home. & $17(30.4)$ \\
\hline I provide home visits for my cancer patients. & $9(16.1)$ \\
\hline I know which drugs my cancer patients use. & $22(39.3)$ \\
\hline I know whether my cancer patients can pay for their treatment cost. & $14(25.0)$ \\
\hline My cancer patients inform me about their treatment process. & $21(37.5)$ \\
\hline I contact specialists for my cancer patients. & $12(21.5)$ \\
\hline I leave enough time for to listen to my cancer patients' cancer-related problems. & $27(48.2)$ \\
\hline My cancer patients feel comfortable when they talk to me about their worries and challenges. & $44(78.6)$ \\
\hline
\end{tabular}

FPs, family physicians; HPV, human papilomavirus. ${ }^{a}$ Multiple choice can be marked.

lead-poisoning incidents. Someone over there is registered to me, but it is far away. It is not possible for me to follow him/her or know that region."

- Interview 14

All the FPs indicated that their health education and screening services were limited to patients who consulted the family health center for any reason and that they could not pay attention to people who did not consult an FP. Nine FPs mentioned some problems regarding the accessibility of screening services for people who had difficulty reaching the family health center, those who had low education levels and those who lived far away from the screening services. Additionally, thirteen FPs stated that most people for whom they recommended screening did not participate because of their ignorance.

"We have their phone numbers, but if you mean 'contact them' in the literal sense of 'to talk them face-to-face, 'then as I said, I have been here for four years, but I know that some registered people never come to the family health center."

- Interview 5
3) The issues with coordination between family physicians and specialists

Almost all the FPs $(n=14)$ stated that they only had access to information regarding a patient's health condition or cancer diagnoses made by specialists when the patient came to the family health center for some reason. All but one of the physicians noted the lack of a referral system caused problems with patient utilization of preventive services and with monitoring patients and keeping records as well as care coordination. They noted that after diagnosis, there was no communication between the FPs and the specialists. Three physicians emphasized the need for a kind of electronic data system that would allow primary, secondary, and tertiary care providers to share data with one another.

"There is no correspondence or any other communication between the specialists and us. Only if they give the patients a piece of paper and tell them, 'Have that test or have those drugs prescribed at the family health center' we only have information about the patient or treatment in this way."

- Interview 10 
4) The effect of the number of primary care team members

All but four of the FPs reported that their workloads were much too high because there was only one family health worker to assist them. Additionally, they stated that they had difficulties carrying out their responsibilities in cancer control and implementing preventive measures in general. The FPs indicated that cancer screening tests, such as taking samples for pap smear and fecal occult blood test, were often conducted by the family health workers. The four FPs emphasized that dissatisfaction was present among the family health workers, who had difficulties carrying out their routine work, and that trouble could be observed among health personnel.

"We cannot save 5-10 minutes per person and give him/her information because many patients are waiting at the door to consult us. As you work too much and do additional work, you forget about our primary duties, such as cancer screening."

— Interview 12

\section{DISCUSSION}

This study provided information on the functions of FPs and an in-depth understanding of their perceptions and challenges regarding cancer control in Karabuk province, Turkey. When interpreting the findings of the study, several limitations should be considered. First, the findings were based only on FPs' perspectives and not those of the community, cancer patients or specialists. Second, the sample size of the quantitative part of the study was small. However, as it included all the FPs in Karabuk Province, the findings represent the entire province. Another important limitation of our study is that it was based on physicians' self-reports, and it is possible that the FPs responded in ways that reflected better practices rather than what they actually did. Finally, although the qualitative part of this study provided a comprehensive and in-depth understanding of the perceptions and experiences of the FPs regarding cancer control, the findings might not be generalizable to all FPs in Karabuk.

In the study, all the FPs stated that their primary responsibilities in cancer control were primary and secondary prevention, but only half the FPs reported that they provided opportunistic health education, and many FPs had not recommended the screenings included in the national cancer screening program to a person in the last three months. Mcilfatrick et al. ${ }^{12}$ reported that $66 \%$ of general practitioners in a region of the United Kingdom routinely provided cancer preven- tion information for the primary risk factors of cancer and for maintaining a healthy lifestyle. That study also indicated that most general physicians (97\%) questioned patients about their smoking status and whether they had received any help to quit smoking, and they promoted screening for cervical cancer.

Cancer care begins with referral and diagnosis and continues through treatment to follow-up care. Care requires considering the patient's physical needs as well as the psychological and social challenges of patients and their families. ${ }^{13}$ However, this study indicated that the functions of FPs almost disappeared after diagnosis. The FPs did not see themselves as primarily responsible for providing follow-up and supportive care for cancer patients, and they did not wish to assume this role. Most of the physicians did not know even the how many cancer patients were in their list, and only $16.1 \%$ of the physicians stated that they visited cancer patients at home. These are important finding that reflects the FPs' approach to managing cancer and other non-communicable diseases. In developed countries, although some problems exist in FPs' involvement in the care of cancer patients after diagnosis, FPs feel responsible for the care of patients and their families and provide routine follow-up care for them. Beccaro et al. ${ }^{4}$ reported that general practitioners needed training on the theoretical issues of palliative care but were very willing to integrate into the multi-professional palliative care team and provide palliative care for their patients in coordination with palliative care physicians in their daily practices. In a survey of 356 general practitioners in London, $61 \%$ were providing palliative care to cancer patients on their list; $72 \%$ agreed or strongly agreed that palliative care was a central part of their role, and only $27 \%$ wanted to hand over the care to specialists. ${ }^{14}$ Anvik et al. ${ }^{8}$ reported that, in North Norway, general practitioners have a place in the follow-up of many patients with cancer and in the initial phase after treatment, and patients trust them to provide competent care, especially when they have more complex health care needs on top of their cancer.

This study showed that FPs provide health education and screening services only to people who visit the family health center, and they do not make efforts to reach non-service-seekers. As the findings of the qualitative part of the study reflect, demand-oriented care causes a decrease in the access and the use of services by people who are socially disadvantaged and have more health service needs. Afterwards, list-based organization removed the description of the community according to its demographic, social, economic, and other characteristics. For this reason, list-based organization causes important problems for FPs in terms of identifying community needs, providing surveillance, and planning and evaluating their services. As the current study 
showed, it is quite difficult to take precautions against risk factors, provide early detection and follow-up patients' care needs without defining the community. ${ }^{15,16}$

As cancer patients move among FPs, hospitals and oncology clinics, continuity of cares requires sharing patient information, coordinating administrative plans, and sharing communication among professionals. Primary care physicians are considered the best professionals to perform these activities. ${ }^{17}$ However, in this study, we found that there was insufficient coordination between the FPs and patients and between the FPs and specialists. The difficulty of coordinating cancer care when patients began receiving specialized services has also been emphasized in other studies. ${ }^{910,17}$ The nature of a cancer diagnosis, the complexity and toxicity of cancer treatments, and the structure of the cancer care system all pose significant challenges to high-quality coordination of care. Norman et al. ${ }^{18}$ reported that cancer patients lose contact with FPs because of patient or physician relocation, distrust over delays in diagnosis, the lack of a perceived need for an FP, poor communication between FPs and specialists, and a lack of FP involvement in the hospital.

In this study, the FPs reported that the lack of a referral system caused problems with patient utilization of preventive services and with monitoring patients and keeping records. The referral system has never been operated since 2004 when the family medicine model began piloting. Limiting the gatekeeping functions of FPs adversely affects their ability to plan care and ensure its continuity, substantially limits their role in allowing patients to die in familiar surroundings and decreases the weight of their decisions as an end-of-life care provider. ${ }^{5}$ Also, Phillips et al. ${ }^{19}$ found that women in gatekeeper plans were significantly more likely to obtain mammography screening, clinical breast examinations and pap smears than women who were not in gatekeeper plans.

Another important finding of the study was that the FPs did not have time to provide preventive services due to their excessive responsibilities and the presence of only one family health worker per family health unit. This problem is also emphasized in other studies from Turkey. ${ }^{20,21}$ The physicians reported that they were able to examine patients and perform other daily duties by limiting the time they used for cancer prevention services and that it was impossible to plan for cancer control services and follow cancer patients because of excessive responsibilities and limited time. It was estimated that a physician would need 1,773 hours per year or 7.4 hours per workday to meet all the US Preventive Task Force suggestions fully. ${ }^{22}$
These findings reflect the need for a multidisciplinary team to treat cancer and other chronic diseases in primary care.

In conclusion, despite the limitations mentioned above, this study identified critical gaps in the provision of cancer control by FPs. The participating FPs reported that primary and secondary prevention activities for cancer were unplanned, opportunistic and insufficient and that they did not take responsibility for their cancer patients' follow-up and palliative care. The study also showed that the inadequate role of FPs in cancer control services was limited not only by their approaches but also by the reformed organization of primary care services. Further comprehensive studies are necessary to identify FPs' role in cancer control and to determine how to better integrate FPs into cancer control to ensure the improvement of preventive services and cancer care in Turkey. Additionally, FPs should receive in-service on the management of cancer and other chronic diseases in the primary care setting, and the responsibilities of FPs should be clearly defined for all stages of cancer control.

\section{ACKNOWLEDGMENTS}

The authors would like to thank the family physicians who participated in the study. We also thank Ayșe Ozkan and Hasan Yildirim for their contribution of the study.

\section{CONFLICTS OF INTEREST}

No potential conflicts of interest were disclosed.

\section{REFERENCES}

1. McIsaac WJ, Fuller-Thomson E, Talbot Y. Does having regular care by a family physician improve preventive care? Can Fam Physician 2001;47:70-6.

2. Lambrew JM, DeFriese GH, Carey TS, Ricketts TC, Biddle AK. The effects of having a regular doctor on access to primary care. Med Care 1996;34:138-51.

3. Miser WF. Cancer screening in the primary care setting: the role of the primary care physician in screening for breast, cervical, colorectal, lung, ovarian, and prostate cancers. Prim Care 2007; 34:137-67.

4. Beccaro M, Lora Aprile P, Scaccabarozzi G, Cancian M, Costantini M. Survey of Italian general practitioners: knowledge, opinions, and activities of palliative care. J Pain Symptom Manage 2013;46: 335-44.

5. Reyniers T, Houttekier D, Pasman HR, Stichele RV, Cohen J, Deliens L. The family physician's perceived role in preventing and guiding hospital admissions at the end of life: a focus group study. Ann Fam Med 2014;12:441-6.

6. Roetzheim RG, Ferrante JM, Lee JH, Chen R, Love-Jackson KM, 
Gonzalez EC, et al. Influence of primary care on breast cancer outcomes among medicare beneficiaries. Ann Fam Med 2012; 10:401-11.

7. Gorey KM, Luginaah IN, Holowaty EJ, Fung KY, Hamm C. Associations of physician supplies with breast cancer stage at diagnosis and survival in Ontario, 1988 to 2006. Cancer 2009;115: 3563-70.

8. Anvik T, Holtedahl KA, Mikalsen H. "When patients have cancer, they stop seeing me"--the role of the general practitioner in early follow-up of patients with cancer--a qualitative study. BMC Fam Pract 2006;7:19.

9. Sisler JJ, Brown JB, Stewart M. Family physicians' roles in cancer care. Survey of patients on a provincial cancer registry. Can Fam Physician 2004:50:889-96.

10. Miedema B, MacDonald I, Tatemichi S. Cancer follow-up care. Patients' perspectives. Can Fam Physician 2003:49:890-5.

11. Republic of Turkey Ministry of Health, Turkey Public Health Institution, Cancer Control Department. Turkey cancer control programme. https:/www.iccp-portal.org/system/files/plans/Turkiye_ Kanser_Kontrol Program English.pdf. Accessed 2016.

12. McIlfatrick S, Keeney S, McKenna H, McCarley N, McElwee G. Investigating the role of the general practitioner in cancer prevention: a mixed methods study. BMC Fam Pract 2013;14:58.

13. Jiwa M, McManus A, Dadich A. Continuity of cancer care: where do primary care practitioners fit in? Cancer Forum 2013:37:31-4.

14. Burt J, Shipman C, White P, Addington-Hall J. Roles, service knowledge and priorities in the provision of palliative care: a postal survey of London GPs. Palliat Med 2006;20:487-92.

15. Burström B. Market-oriented, demand-driven health care reforms and equity in health and health care utilization in Sweden. Int J Health Serv 2009;39:271-85.

16. Burström B, Burström K, Nilsson G, Tomson G, Whitehead M, Winblad U. Equity aspects of the primary health care choice reform in Sweden: a scoping review. Int J Equity Health 2017; $16: 29$.

17. Sisler J, McCormack-Speak P. Bridging the gap between primary care and the cancer system: the UPCON network of CancerCare Manitoba. Can Fam Physician 2009:55:273-8.

18. Norman A, Sisler J, Hack T, Harlos M. Family physicians and cancer care. Palliative care patients' perspectives. Can Fam Physician 2001;47:2009-12, 2015-6.

19. Phillips KA, Haas JS, Liang SY, Baker LC, Tye S, Kerlikowske K, et al. Are gatekeeper requirements associated with cancer screening utilization? Health Serv Res 2004:39:153-78.

20. Çiçeklioğlu M, Öcek ZA, Turk M, Taner Ş. The influence of a market-oriented primary care reform on family physicians working conditions: a qualitative study in Turkey. Eur J Gen Pract 2015; 21:97-102.

21. Öcek ZA, Çiçeklioğlu M, Yücel U, Özdemir R. Family medicine model in Turkey: a qualitative assessment from the perspectives of primary care workers. BMC Fam Pract 2014;15:38.

22. Yarnall KS, Pollak KI, Østbye T, Krause KM, Michener JL. Primary care: is there enough time for prevention? Am J Public Health 2003:93:635-41. 